\title{
Nontraumatic Descemet Membrane Detachment with Tear in Osteogenesis Imperfecta
}

\author{
Nihat Polat $\cdot$ Pamuk Betül Ulucan
}

To view enhanced content go to www.ophthalmology-open.com

Received: February 5, 2015 / Published online: April 9, 2015

(c) The Author(s) 2015. This article is published with open access at Springerlink.com

\section{ABSTRACT}

We report the first case of unilateral spontaneous Descemet membrane detachment (DMD) with tear occurring in a patient with osteogenesis imperfecta (OI). A 20-year-old male patient with OI presented with a history of recent primary repair ( 2 weeks prior) of left globe rupture following local finger trauma to the left eye. The patient had no history of other ocular surgery or trauma. The examination revealed a best corrected visual acuity of 20/40 in the right and no light perception in the left eye. Slit-lamp examination showed an oval giant Descemet tear extending from the 12 o'clock to the 5 o'clock area and a large DMD involving the upper and nasal quadrants in the right cornea. It was thought that monitoring the patient without intervention and only considering a surgical procedure if the disorder progressed was the best option, taking into

Electronic supplementary material The online version of this article (doi:10.1007/s40123-015-0034-2) contains supplementary material, which is available to authorized users.

N. Polat $(\bowtie) \cdot$ P. B. Ulucan

Department of Ophthalmology, Medical Faculty, Inonu University, 44280 Malatya, Turkey

e-mail: drnihatpolat@gmail.com account the patient's reasonable visual acuity and the risks of keratoplasty. The dimensions of the DMD and tear had remained the same at 1-year follow-up period. We believe that followup without intervention should be considered for non-progressive DMD with a giant tear if the patient has a single functional eye.

Keywords: Cornea; Descemet membrane detachment (DMD); Osteogenesis imperfecta (OI)

\section{INTRODUCTION}

Osteogenesis imperfecta (OI) is a connective tissue disorder of the skeleton, ears, eyes, teeth, skin and joints, caused by an abnormality of type I collagen [1]. OI is generally inherited in an autosomal dominant fashion and is caused by mutations in the genes that code for type I procollagen (Collagen, type 1, alpha 1 and Collagen, type 1, alpha 2) [2]. Ocular features of OI include megalocornea, keratoconus, anterior embryotoxon, hyperopia, blue sclera (secondary to reduced scleral thickness), spontaneous globe rupture, primary optic 
atrophy, optic atrophy secondary to chronic papilledema, papilledema, congenital glaucoma, zonular cataracts, dislocated lens, choroidal sclerosis, retinal and subhyaloid hemorrhage, and partial color blindness [3]. Descemet membrane detachments (DMDs) occur when there is a separation of the endothelium-Descemet membrane complex from the posterior corneal stroma. They can result in corneal edema, double anterior chamber, decreased vision and corneal decompensation. DMD has been reported to occur after various intraocular surgeries, alkaline injuries or even spontaneously in predisposed eyes [4].

We report the first case of follow-up without intervention of a patient with unilateral spontaneous DMD with tear in an OI.

\section{CASE}

A 20-year-old male patient with OI presented with a history of recent primary repair of left globe rupture ( 2 weeks prior) following local finger trauma to the left eye. The patient had no history of other ocular surgery. The examination revealed a best corrected visual acuity (BCVA) of 20/40 in the right eye and no light perception in the left eye. Slit-lamp examination showed an oval giant Descemet tear extending from the $12 \mathrm{o}^{\prime}$ clock to the 5 o'clock area and a large DMD involving the upper and nasal quadrants in the right cornea (Fig. 1). The patient had no other ocular clinical features of OI. The DMD was observed to be stretched (Fig. 2). There was no corneal edema, but interestingly there was peripheral superficial corneal vascularization in the areas where the Descemet membrane was attached. There was no history of contact lens use. The iris, lens and fundus were normal. The intraocular pressure

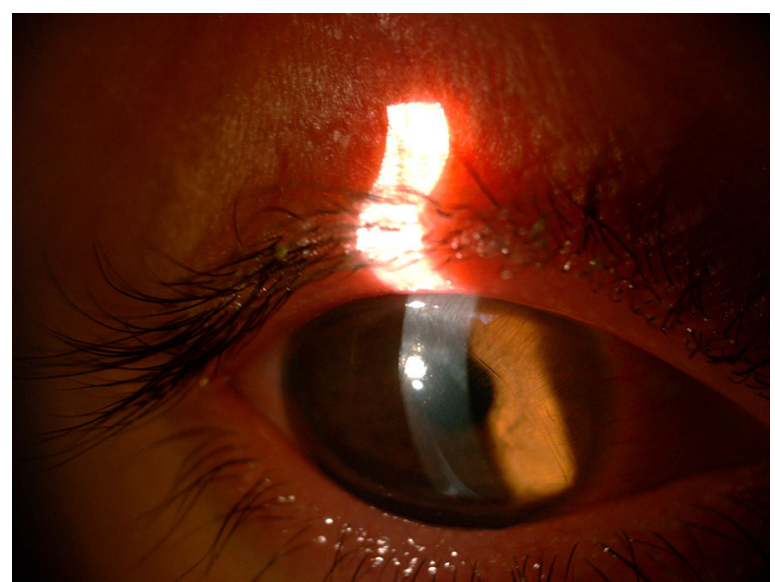

Fig. 1 The image of the right showing Descemet tear

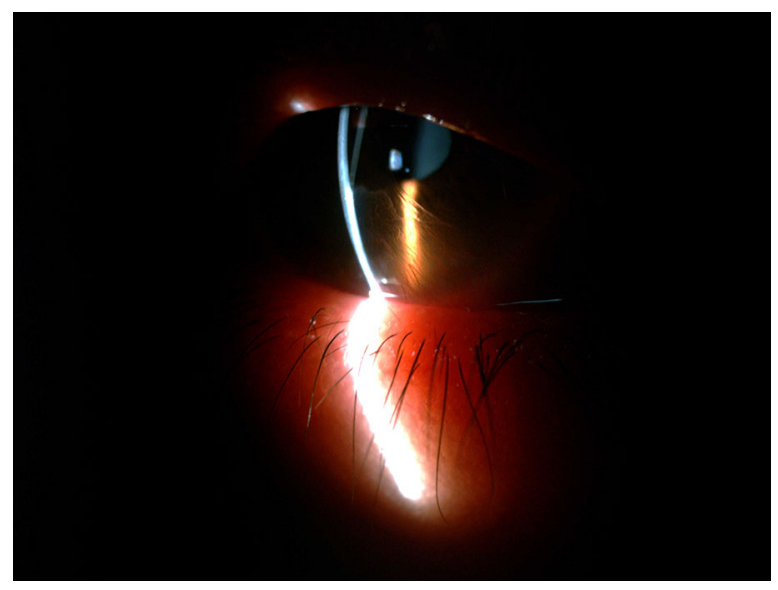

Fig. 2 Slit-lamp image of the right eye showing Descemet membrane detachment

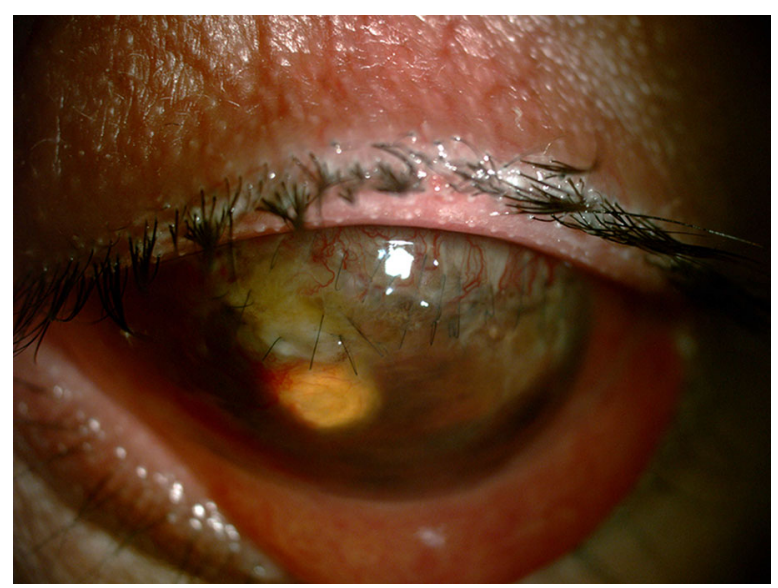

Fig. 3 Image of the left eye showing vascularization of the peripheral cornea similar to the right eye. The conjunctiva was hyperemic and chemotic 


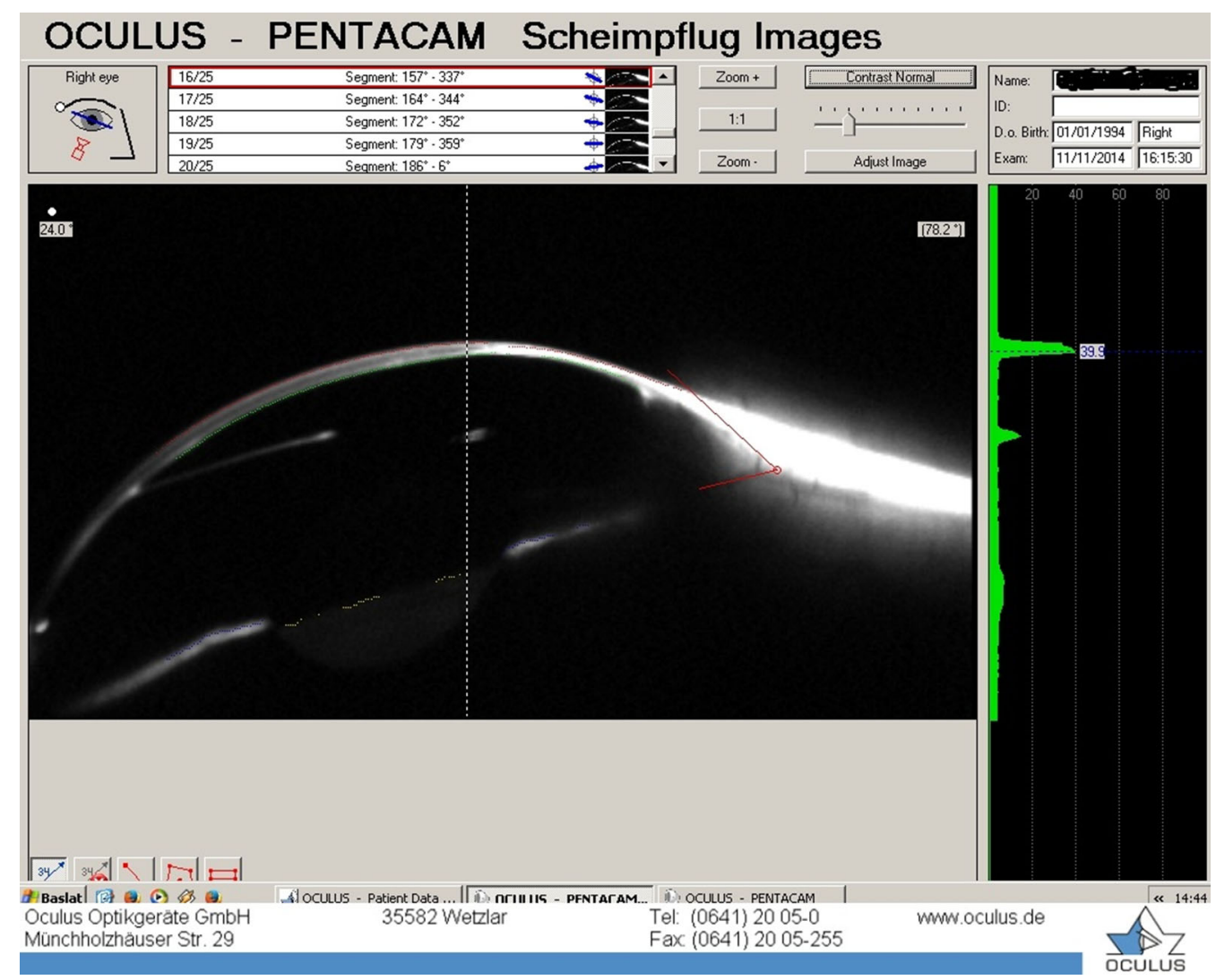

Fig. 4 Scheimpflug image of the right eye showing osteogenesis imperfecta and Descemet membrane detachment

was $20 \mathrm{mmHg}$ when measured with a Goldmann applanation tonometer. The left eye had no light perception during the postoperative period. The anterior chamber structures could not be discerned on the slit lamp due to hyphema but the perforated areas had been sutured. The peripheral cornea of the left eye showed vascularization similar to the right eye. The conjunctiva was hyperemic and chemotic (Fig. 3).

A Scheimpflug image of the right eye confirmed DMD with tear (Fig. 4). There were no symptoms in the right eye other than significant photosensitivity. The patient was advised to wear sunglasses at all times and was followed up without any medical treatment or surgical intervention for the right eye. He was advised to stay away from activities that could lead to trauma. There was no change in symptoms or ocular features at the 1-month follow up. The dimensions of the DMD with tear had remained the same. BCVA of right eye was 20/40, intraocular pressure was $20 \mathrm{mmHg}$, and the dimensions of the DMD and tear had remained unchanged at 1-year follow-up. The patient was followed up at 3-month intervals and with the same recommendations.

Informed consent was obtained from all patients for being included in the study.

\section{DISCUSSION}

The patient had a history of traumatic left globe perforation together with a right nontraumatic DMD. DMD is a recognized problem in patients with OI, and it is possible that a collagen 
structure defect created a predisposition for this condition in this patient [3]. What made this case interesting was the presence of a DMD with tear. This is the first reported case that has developed without trauma, but DMDs have also been reported to occur 'spontaneously' as hydrops events in ectatic corneas [5]. Another interesting point of this case was that visual acuity had not significantly decreased, despite the occurrence of light sensitivity. In addition corneal edema did not occur, although the DMD affected almost half the cornea in one eye. Corneal clearance, despite the persistence of DMD, has also been reported [6]. This has been attributed to endothelial cell hypertrophy, migration, redistribution and/or repopulation [7]. To date, no prospective or comparative studies on the management options for DMDs have been published. The choice to adopt conservative versus surgical treatment for DMD is largely based on results from retrospective case series, anecdotal reports, surgeon's preference and patient choice [4]. Most DMDs remain small and localized to the wound but some patients present with large, extensive detachments that result in severe corneal edema and a marked reduction in visual acuity. Traditional treatment regimens have included observation, intracameral injections of air, or viscoelastic, transcorneal suturing, and even corneal transplantation. Intracameral sulfur hexafluoride or perfluoropropane gas injections have gained increasing acceptance as an efficient and effective treatment option for DMD in recent years [8].

In this patient, the left eye had lost its function, despite repair following globe rupture, and the patient would therefore be subsequently considered to have a single eye. Interventions scheduled to be performed on the right eye had therefore become even more important. Intraocular air injection or transcorneal suturing could increase DMD with tear as the Descemet membrane was already observed to be stretched. It was thought that monitoring the patient without intervention and considering a surgical procedure only if the disorder progressed was the best option, taking into account the patient's reasonable visual acuity and the risks of keratoplasty. The patient continues to be followed up without any intervention as there has been no progress of the disorder.

\section{CONCLUSION}

In conclusion, DMD with tear can develop without trauma in the eyes of patients with predisposing disorders such as OI. In similar cases it would be recommended to take the patient's visual acuity, type of detachment, whether the disorder is progressive, the other eye's status and the risks of the surgical procedures into consideration when deciding on the course of action.

\section{ACKNOWLEDGMENTS}

There were no substantial contributions provided by any individuals other than the stated authors. No funding or sponsorship was received for this study or publication of this article. All named authors meet the International Committee of Medical Journal Editors (ICMJE) criteria for authorship for this manuscript, take responsibility for the integrity of the work as a whole, and have given final approval for the version to be published.

Conflict of interest. N. Polat and P. B. Ulucan declare no conflict of interest or financial support. 
Compliance with ethics guidelines. İnformed consent was obtained from the patient for being included in the study.

Open Access. This article is distributed under the terms of the Creative Commons Attribution Noncommercial License which permits any noncommercial use, distribution, and reproduction in any medium, provided the original author(s) and the source are credited.

\section{REFERENCES}

1. Byers PH, Cole WG. Osteogenesis imperfect. In: Royce BM, Steinmann B, editors. Connective tissue and its heritable disorders: molecular, genetic and medical aspects. New York: Wiley-Liss; 1993. p. 385-430.

2. Esposito P, Plotkin H. Surgical treatment of osteogenesis imperfecta: current concepts. Curr Opin Pediatr. 2008;20:52-7.

3. Gorovoy MS, Gorovoy IR, Ullman S, Gorovoy JB. Descemet stripping automated endothelial keratoplasty for spontaneous descemet membrane detachment in a patient with osteogenesis imperfecta. Cornea. 2012;31:832-5.

4. Chow VW, Agarwal T, Vajpayee RB, Jhanji V. Update on diagnosis and management of Descemet's membrane detachment. Curr Opin Ophthalmol. 2013;24:356-61.

5. Nakagawa T, Maeda N, Okazaki N, Hori Y, Nishida K, Tano Y. Ultrasound biomicroscopic examination of acute hydrops in patients with keratoconus. Am J Ophthalmol. 2006;141:1134-6.

6. Kolomeyer AM, Chu DS. Descemet stripping endothelial keratoplasty in a patient with keratoglobus and chronic hydrops secondary to a spontaneous descemet membrane tear. Case Rep Ophthalmol Med. 2013:ID 697403, p. 5.

7. Watson SL, Abiad G, Coroneo MT. Spontaneous resolution of corneal oedema following Descemet's detachment. Clin Experiment Ophthalmol. 2006;34:797-9.

8. Kim T, Hasan SA. A new technique for repairing Descemet's membrane detachments using intracameral gas injection. Arch Ophthalmol. 2002;120:181-3. 